\title{
Study on Phenolic Resins Modified by Copper Nanoparticles
}

\author{
Ronghui Lin,,${ }^{1,2}$ Liang FANG, ${ }^{1}$ Xinping LI, ${ }^{3}$ Yingxin XI, ${ }^{4,}$ Sufeng ZHANG,${ }^{3}$ and Ping SuN ${ }^{2}$ \\ ${ }^{1}$ School of Materials Science and Engineering, Xi'an Jiaotong University, Xi'an 710049, P. R. China \\ ${ }^{2}$ School of Mechanical Engineering, Qingdao Technological University, Qingdao 266033, P. R. China \\ ${ }^{3}$ School of Paper Making Engineering, Shan Xi University of Science and Technology, \\ Xian Yang 712081, P. R. China \\ ${ }^{4}$ School of Science, Xi'an Jiaotong University, Xi'an 710049, P. R. China
}

(Received June 20, 2005; Accepted October 17, 2005; Published February 15, 2006)

\begin{abstract}
Phenol-formaldehyde (PF) resins modified by nanosized copper particles were synthesized by in situ polymerization process. XRD, TEM revealed that the copper particles in the resulting PF resins had a spherical geometry with a measurement of 30-60 $\mathrm{nm}$ in diameter, and there were few which were agglomerate. The thermal properties of the prepared PF resins were investigated by thermogravimetric analysis (TGA). It was indicated that copper nanoparticles remarkably improved the thermal stability of the PF resins at lower temperature. The initial decomposition temperature of the modified PF resin could increase by $47^{\circ} \mathrm{C}$, compared to the pure one. However, the copper nanoparticles increased the rate of the degradation of the PF resins at the elevated temperature. The effects of copper nanoparticles on the thermal properties of the PF resins, which were used as a matrix of a friction material, were needed. The toughness of the prepared PF resins was also studied. The results revealed that copper nanoparticles obviously improved the brittleness of the PF resins. The impact strength of the modified PF resin might increase by $66.6 \%$, compared to the pure one. [DOI 10.1295/polymj.38.178]

KEY WORDS Phenol-formaldehyde Resin / Copper Nanoparticle / Impact Strength / Thermal Stability /
\end{abstract}

Phenol-formaldehyde $(\mathrm{PF})$ resins have been and are still being used extensively in friction materials (FM) as a matrix. The properties of PF resins directly affect the heat resistance and friction and wear behaviors of FM. Although conventional PF resins possess excellent dimensional stability, structural integrity, solvent resistance, and so forth, ${ }^{1}$ some of the properties of the $\mathrm{PF}$ resins need further improvement in order to meet the ever-increasing performance requirements of modern FM. The adverse properties, which require research attention, mainly include inherent brittleness and moderate thermal-oxidative stability. In order to improve these properties, many substances, such as molybdenum, boron, tung oil, cashew nut shell oil, rubber, etc. have been chosen to modify the PF resins. ${ }^{2-4}$ However, in terms of increasing both toughness and heat resistance, any one of the substances mentioned above has not been satisfactory.

In recent years, nanosized particle modified PF resins have stimulated great interest in many researchers due to their unique properties, such as high thermal resistance, high tensile strength and good toughness. ${ }^{5}$ The appearance of nano-technology opens a new route for improving the PF resins. Copper is one of the fillings often used in FM. Copper can significantly improve friction and wear performances and thermal conductivity of FM. Therefore, it would be presumed that the PF resins modified by nanosized copper particles should exhibit significant improvements in toughness, thermal resistance, and friction and wear performances. However, to the best of our knowledge, the PF resins modified by copper nanoparticles have not been studied yet.

In this paper, we focus on the synthesis of the PF resins modified by copper nanoparticles using in situ polymerization process and on the examination of the thermal and mechanical properties of them.

\section{EXPERIMENTAL}

\section{Materials}

Phenol and formaldehyde monomer were obtained from Jinshan chemical reagent plant, Chendu, China. Tartaric acid, disodium ethylene diamine tatra-acetate (EDTA-2Na), and copper sulphate $\left(\mathrm{CuSO}_{4}\right)$ were analytical reagents obtained from Dengfeng chemical reagent plant, Tianjing, China. Poly vinyl pyrrolidone (PVP) and potassium boron hydride were analytical reagents purchased from Shanghai Chemical Reagent Co., China. Twelve alkyl sodium sulphate, hydroxyl ethyl cellulose (HEC) and sodium pyrophosphate were purified industry-grade reagents supplied by Xian Chemical reagent Co., China. 


\section{Preparation of Nanosized Copper Particles}

The preparation of the copper nanoparticles involved three solutions, A, B and C. $600 \mathrm{~mL}$ of solution A consisted of $\mathrm{CuSO}_{4}(0.15 \mathrm{~mol})$, tartaric acid $(0.6$ mol), $\mathrm{NaOH}(1.85 \mathrm{~mol})$, and $\mathrm{H}_{2} \mathrm{O}$ (33 mol); $720 \mathrm{~mL}$ of solution $\mathrm{B}$ contained $\mathrm{KBH}_{4}(0.36 \mathrm{~mol})$, sodium pyrophosphate $(0.03 \mathrm{~mol})$, sodium alginate $(2.16 \mathrm{~g})$, HEC $(8.63 \mathrm{~g}), \mathrm{NaOH}(0.21 \mathrm{~mol})$ and $\mathrm{H}_{2} \mathrm{O}(40 \mathrm{~mol}) ; 60 \mathrm{~mL}$ of solution C composed of PVP (1.3 g), SDS (1.66 g) and $\mathrm{H}_{2} \mathrm{O}$ (3.3 mol). Solution $\mathrm{C}$ was added to solution $\mathrm{B}$ with stirring to make a reducing solution containing stabilizing agents for colloids, to which solution A was then added dropwise under vigorous stirring. The resulting mixture was stirred continuously for another $3 \mathrm{~h}$ to form a red brown colloidal solution. After the colloid was centrifuged and washed two times with distilled water, the obtained particles were then dissolved in a solution containing $1 \mathrm{wt} \%$ ammonium polyacrylate to make a copper colloid using a sonicator. All the operations above were at room temperature. The copper colloid was kept in a sealed bottle for preparation of the modified $\mathrm{PF}$ resins.

In our route, the reaction of $\mathrm{Cu}^{2+}$ with $\mathrm{BH}_{4}{ }^{-}$under basic conditions may take place as follows: ${ }^{6}$

$$
\begin{gathered}
\mathrm{Cu}^{2+}+2 \mathrm{BH}_{4}^{-}+2 \mathrm{OH}^{-}+2 \mathrm{H}_{2} \mathrm{O} \\
\rightarrow \mathrm{Cu}+2 \mathrm{BO}_{2}^{-}+7 \mathrm{H}_{2}
\end{gathered}
$$

\section{Preparation of PF Resins Modified by Nanosized Cop- per Particles}

In our route, the process of the preparation of the $\mathrm{PF}$ resins modified by copper nanoparticles was divided into three steps. Step 1, phenol reacted with formaldehyde to produce a prepolymer of low viscosity. Step 2, the prepolymer mixed with copper colloid. Step 3, the resulting mixture polymerized.

The modified PF resins were synthesized in a $0.5 \mathrm{~L}$ glass reactor equipped with a thermometer, a reflux condenser and a stirrer. The reagents, $42.4 \mathrm{~g}$ phenol $(0.45 \mathrm{~mol})$ and $73 \mathrm{~g}$ formalin (in $37 \% \mathrm{wt} \%$ water solution, $0.9 \mathrm{~mol}$ ), were fed into a flask reactor. The reaction of phenol with formaldehyde proceeded at 90$95^{\circ} \mathrm{C}$ under stirring for $30 \mathrm{~min}$ in the reactor, catalyzed by $\mathrm{NaOH}(2 \mathrm{~g}$ of $\mathrm{NaOH}$ dissolved in $10 \mathrm{~mL}$ of water). Then, it was rapidly stopped by putting the reactor in a cold water bath. According to the desired weight ratio of copper nanoparticles and $\mathrm{PF}$ resin, the fresh copper colloid prepared above was dropwise added into the reactor with vigorously stirring at room temperature. It was followed that the resulting mixtures were heated at $80^{\circ} \mathrm{C}$ under stirring for $50 \mathrm{~min}$ to polymerize and then rapidly cooled to room temperature, namely getting the PF resin containing copper nanoparticles. The mixing weight ratios of nanosized copper particles and PF resin, which were selected in the study, were $0.6 / 100,1.2 / 100,1.8 / 100$, $2.4 / 100(\mathrm{w} / \mathrm{w} \%)$, and $3 / 100(\mathrm{w} / \mathrm{w} \%)$, respectively. In order to compare the modified $\mathrm{PF}$ resin with the pure one, some pure PF resin was prepared under the same conditions as above.

For X-ray diffraction analysis (XRD) and thermal property experiments, part of the resulting $\mathrm{PF}$ resins were dehydrated at $65^{\circ} \mathrm{C}$ under a vacuum of $67 \mathrm{KPa}$ for $1 \mathrm{~h}$, then cured at $150^{\circ} \mathrm{C}$ for $8 \mathrm{~h}$, and then grinded into powder with 100 micron in diameter.

\section{Measurements}

XRD was carried out on a Rigaku D/max 2400 diffractometer using $\mathrm{CuK} \alpha$ radiation $(\lambda=0.154 \mathrm{~nm})$.

Transmission electron microscopy (TEM) was conducted on a Hitachi H-800 transmission electron microscope using an acceleration voltage of $100 \mathrm{KV}$. A magnification of 100,000 was used.

The size distribution of copper particles was determined with a Mastersizer 2000 laser granulometer.

Thermal degradation of the PF resins was measured using a thermogravimetric analyzer (Dupont-951) from room temperature to $700{ }^{\circ} \mathrm{C}$ with a heating rate of $10^{\circ} \mathrm{C} / \mathrm{min}$ in air.

The charpy impact strength of nonnotched specimens with dimensions $80 \times 10 \times 4 \mathrm{~mm}$ was measured on a simple supported beam impact-testing machine (XJJ-5J) according to the China government standard GB/T 1043-93. The samples for impact strength experiment were prepared by cutting the pasteboard, made from cotton and asbestos, into $85 \mathrm{~mm} \times 12 \mathrm{~mm}$ $\times 4 \mathrm{~mm}$ sheets, then the sheets were immersed in the synthesized $\mathrm{PF}$ resin solutions at $50{ }^{\circ} \mathrm{C}$ for $20 \mathrm{~min}$. The sheets impregnated with the $\mathrm{PF}$ resins were put into a vented oven at $150{ }^{\circ} \mathrm{C}$ for $8 \mathrm{~h}$ to be cured, and then machined to the desired dimensions.

\section{RESULTS AND DISCUSSION}

\section{Characterization}

The X-ray diffraction pattern of the pure PF resin (a) and the modified one with $2.4 \mathrm{wt} \%$ nanosized copper particles (b) are illustrated in Figure 1.

Figure 1a exhibits the big diffraction peak of the pure PF resin. In Comparison with Figure 1a, five new peaks are added in Figure $1 \mathrm{~b}$. The three peaks at $2 \theta=43^{\circ}, 51^{\circ}, 74^{\circ}$, respectively, are attributed to the [111], [200] and [220] reflections of the crystalline copper, indicating the resulting modified PF resin contain simple copper. These peaks broaden, which might result from fine particles and lattice strain. ${ }^{7}$ The rest two peaks, which are weak, are attributed to the [111] and [220] reflections of $\mathrm{Cu}_{2} \mathrm{O}$, indicating the resulting modified $\mathrm{PF}$ resin containing a little amount of $\mathrm{Cu}_{2} \mathrm{O}$. This is due to the oxidation of part of the 

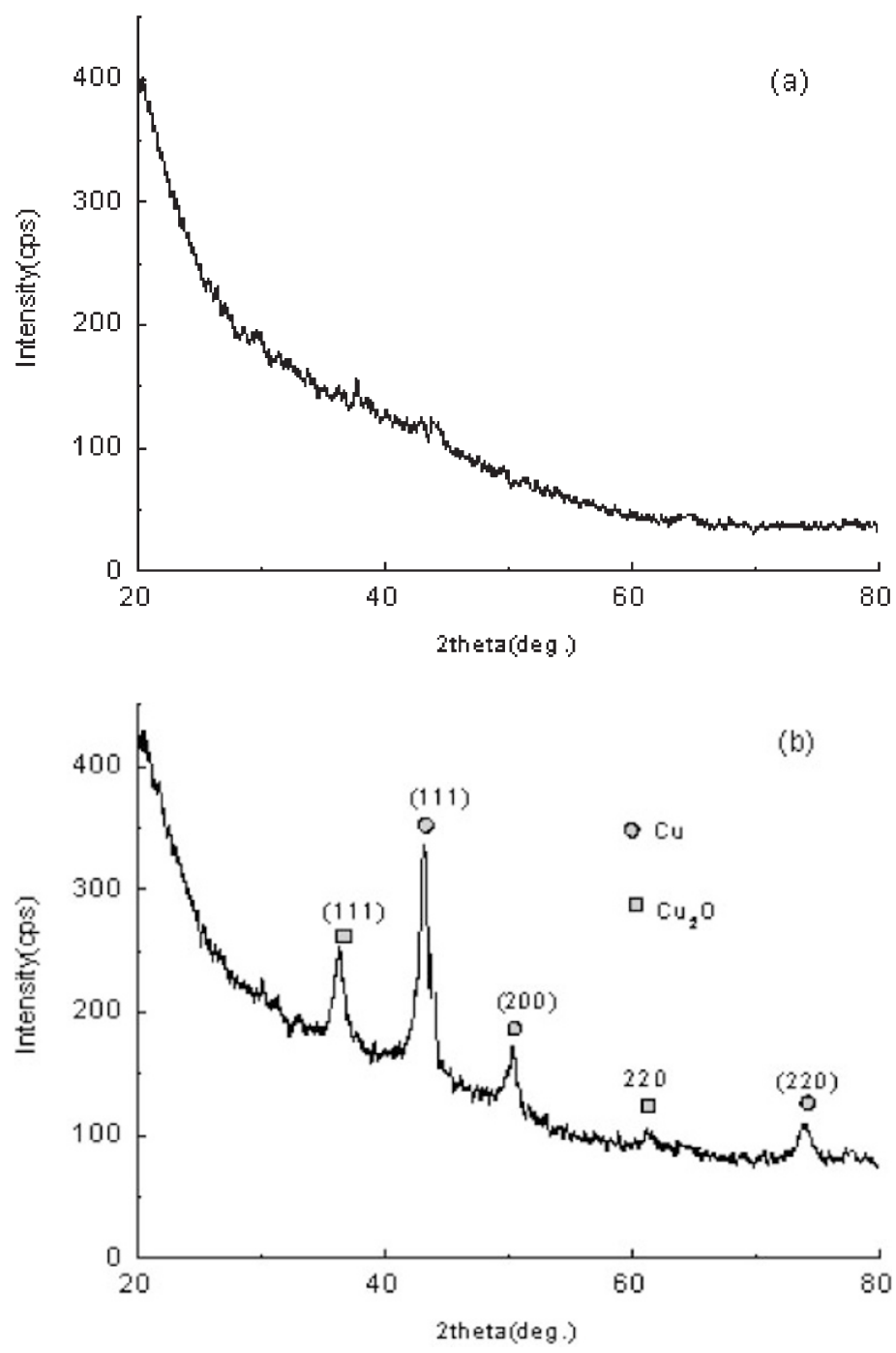

Figure 1. XRD Patterns of the pure PF resin (a) and the modified one with $2.4 \mathrm{wt} \%$ copper nanoparticles (b).

copper particles during the preparation of the samples.

Figure 2 shows the TEM image of the modified PF resin with $2.4 \mathrm{wt} \%$ copper nanoparticles. The black parts in Figure 2 represent copper nanoparticles. It can be seen that the nanosized copper particles are spherical in geometry and dispersed well throughout the PF resin matrix.

The size distribution of the copper particles in the modified PF resins is displayed in Figure 3. Based on Figure 3, it is determinated that the copper particles mainly range from $30-60 \mathrm{~nm}$ in diameter.

In the present study, it is when the prepolymer is in low viscosity that the fresh copper colloid is dropwise added into it. Therefore, the copper nanoparticles can be easily and uniformly dispersed into the PF resin.

The molecular chains of the PF resin have a "space position resistance" effect on the copper nanoparticles, and the effect increases with the length and degree of crosslinking of the molecular chains. In addition, the compound surface active agents, PVP, HEC and ammonium polyacrylate, are applied in the pres-

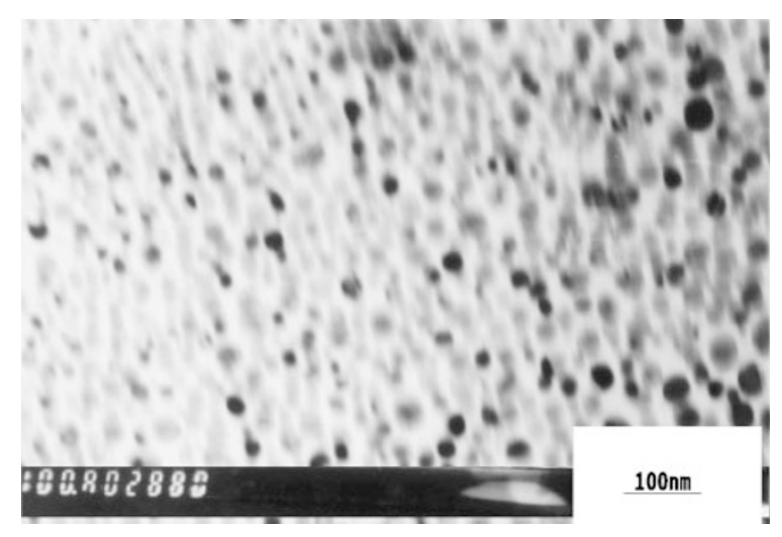

Figure 2. TEM image of the modified PF resin with $2.4 \mathrm{wt} \%$ copper nanoparticles.

ent study. As a result, few of the copper nanoparticles in the modified PF resin agglomerate.

\section{A Physical Model}

The prepared copper particles are too little, only 


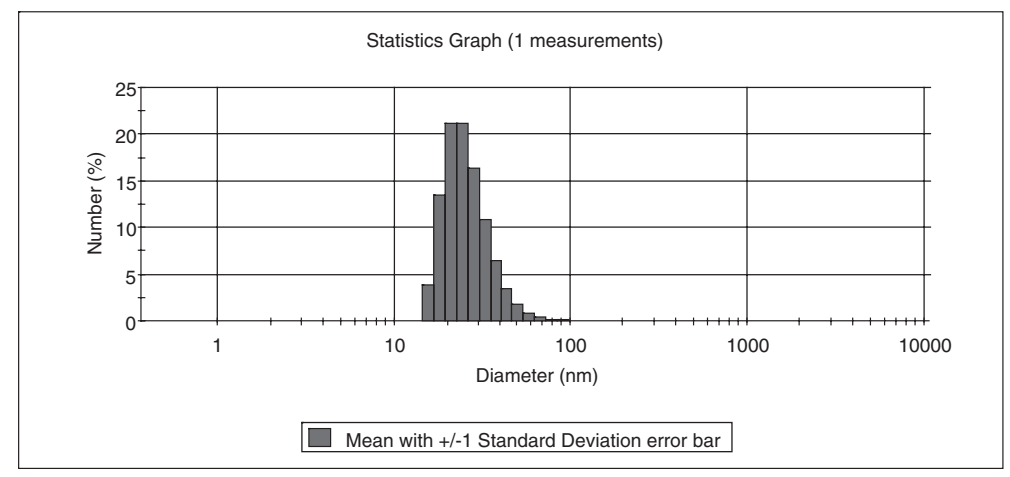

Figure 3. Size distribution of the copper particles in the modified PF resin.

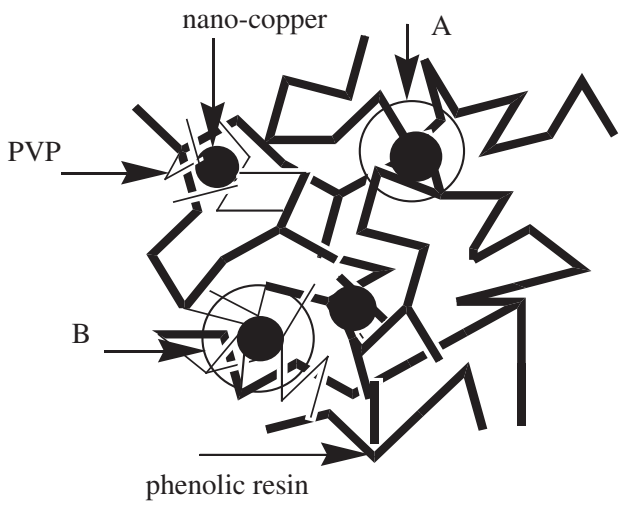

Figure 4. Schematic illustration of the model of interfacial interaction between PF resin and copper nanoparticles.

$30-60 \mathrm{~nm}$ in diameter. They have huge specific surface area, and many unpaired electrons exist on their surfaces. Therefore, they have very high surface energy and chemical activity. When copper nanoparticles are incorporated into PF resin, part of the copper nanoparticles may directly attached to the molecular chains of the PF resin through very strong physical and chemical forces, including van der Waals forces, adsorption and coordinate bonds ${ }^{8}$ (manner A in Figure 4). The others which have been covered by PVP may indirectly attached to the molecular chains of the PF resin through tangle forces between the PF resin and the PVP (manner B in Figure 4). The copper particles dispersed into the PF resin matrix like "anchor points" which firmly link the molecular chains of the PF resin together around them. The model of interfacial interaction between the PF resin and copper nanoparticles is illustrated in Figure 4.

\section{Thermogravimetry}

Figure 5 plots the TGA curves of the prepared PF resins from room temperature to $700^{\circ} \mathrm{C}$ in air. Weight loss at sample A appears in two steps. The first step occurs at $130{ }^{\circ} \mathrm{C}$. In this stage mainly $\mathrm{H}_{2} \mathrm{O}$ evolves. After the initial weight loss of about 5\%, the sample does not lose weight till $305^{\circ} \mathrm{C}$. This stabilization can be explained by the fact that new structures can be formed during oxidation. These structures are stable until the temperature is high enough and the whole polymer structure collapses. ${ }^{9}$ The second weight loss at $305^{\circ} \mathrm{C}$ is caused by oxidative resin degradation. The thermal behaviors of sample C, D, E and F are similar and differ from that of the sample A. Their first weight losses are not evident. This is probably due to the following reasons: firstly, the formation of oxidic layers; secondly, the increase in the degree of crosslinking of the modified PF resins, resulting in a decrease in the amount of $\mathrm{H}_{2} \mathrm{O}$ and other small molecules (such as formaldehyde etc.) in the modified $\mathrm{PF}$ resins. Their final weight losses occur at higher temperatures when compared to sample A. The thermal behaviors of Sample B are similar to that of sample A at temperature below initial decomposition temperature $\left(T_{\mathrm{d}}\right)$, this can be connected with quantity of copper nanoparticles.

The thermograms of sample B, C, D, E and F descend faster than that of sample $\mathrm{A}$ at temperatures above the $T_{\mathrm{d}}$, indicating that the nanosized copper particles significantly influence the degradation process of the PF resins.

Table I shows the $T_{\mathrm{d}}$ of the prepared PF resins. It can be seen that with the increase of copper nanoparticle content, the $T_{\mathrm{d}}$ increases first, and then decreases, when the content is up to $1.2 \mathrm{wt} \%$, it reaches its maximum value and increases by $47^{\circ} \mathrm{C}$ compared to the pure PF resin.

Owing to the "anchor points" effect, the rigidity of the molecular chains of the modified PF resins increases, resulting in an increase in the the flowing resistance and fracture energy of the molecular chains. In addition, copper has good heat conduction and high thermal capacity. It can reduce the temperature gradient in the modified PF resins and absorb heat energy. Consequently, the degradation process of the modified $\mathrm{PF}$ resin is postponed, and its $T_{\mathrm{d}}$ increase. As the content of nanosized copper particles increases, the "anchor points" effect also increases, so the $T_{\mathrm{d}}$ increase. 


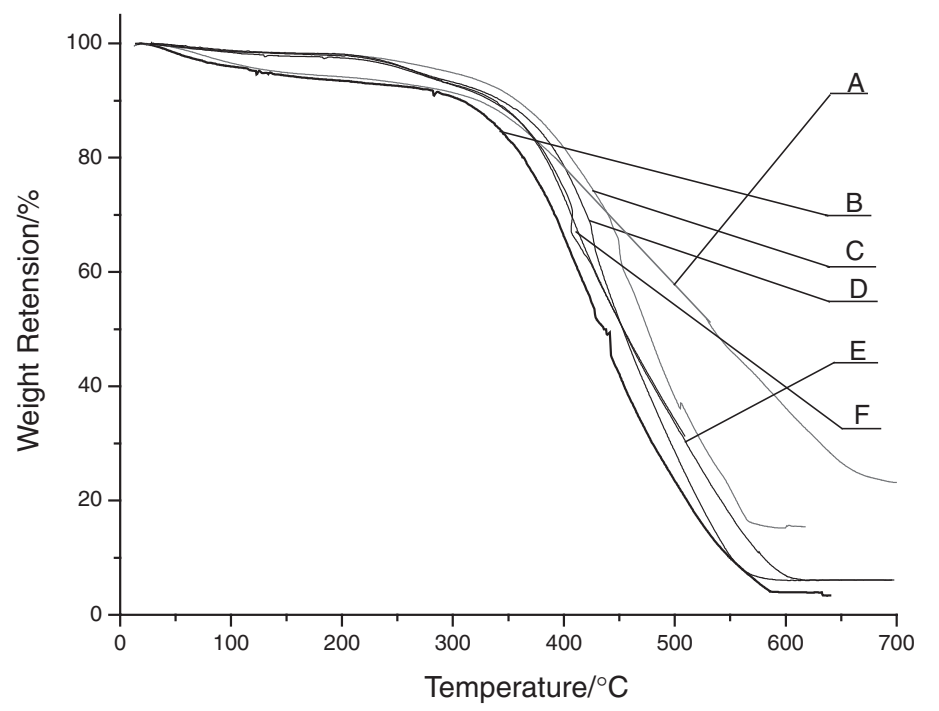

Figure 5. TGA thermograms of the pure $\mathrm{PF}$ resin $(\mathrm{A})$ and the modified ones $(\mathrm{B}-\mathrm{PF}$ resin $+0.6 \% \mathrm{Cu}, \mathrm{C}-\mathrm{PF}$ resin $+1.2 \% \mathrm{Cu}, \mathrm{D}-\mathrm{PF}$ resin $+1.8 \% \mathrm{Cu}, \mathrm{E}-\mathrm{PF}$ resin $+2.4 \% \mathrm{Cu}, \mathrm{F}-\mathrm{PF}$ resin $+3 \% \mathrm{Cu}$ ).

Table I. Initial decomposition temperature of the prepared PF resins

\begin{tabular}{lrrrrrr}
\hline $\begin{array}{l}\text { Copper nanoparticle } \\
\text { content wt } \%\end{array}$ & 0 & 0.6 & 1.2 & 1.8 & 2.4 & 3 \\
\hline $\begin{array}{l}\text { Initial decomposition } \\
\text { temperature } T_{\mathrm{d}}\left({ }^{\circ} \mathrm{C}\right)\end{array}$ & 325 & 342 & 372 & 368 & 363 & 356 \\
\hline
\end{tabular}

However, after the content increases above a critical value, due to the increase in the aggregation of copper nanoparticles, the effective number of "anchor points" decreases, therefore the $T_{\mathrm{d}}$ can not increase further, instead decrease.

The copper nanoparticles have a vast specific surface area and various structural defects on their surface, dramatically enhancing their catalytic activity. ${ }^{10}$ Therefore, copper nanoparticles may act as catalysts to increase the rate of the degradation of the PF resin at elevated temperature. ${ }^{11}$

In some actual applications, especially friction materials, the effects of copper nanoparticles on the thermal properties of PF resins are needed. On one hand, the copper nanoparticles can increase the $T_{\mathrm{d}}$ of PF resin which are used as a matrix of a friction material, thereby increasing the working temperature of the friction material. On the other hand, in case the heat degradation of the friction material has happened, the PF resin in the friction material can rapidly degrade, thereby making an old friction layer be quickly worn off to renew the friction coefficent of the friction material.

\section{Toughness}

Figure 6 shows the impact strength of PF resins as a function of copper nanoparticle content. It is apparent from Figure 5 that with increasing the content of

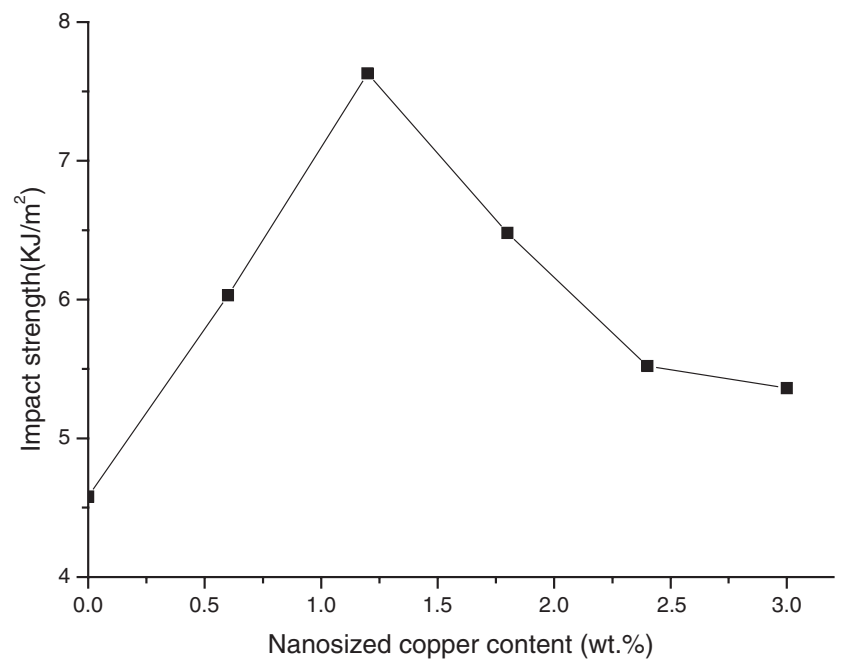

Figure 6. Impact strength curve of $\mathrm{PF}$ resin as a function of copper nanoparticle content.

nanosized copper particles, the impact strength of the modified PF resins increase. When the content is $1.2 \mathrm{wt} \%$, the impact strength is up to its maximum value, increasing by $66.6 \%$ compared to the pure $\mathrm{PF}$ resin. After that, the impact strength falls. The results indicate that by adding a small amount of copper nanoparticles to PF resin, the toughness of PF resin can significantly be improved. Moreover, the excessive addition of nanosized copper particles can not further improve the toughness of PF resin, instead deteriorate it.

Owing to the existence of a great lot of copper nanoparticles which are dispersed well in the PF resin matrix, when PF resin/copper nanocomposites are acted by outer forces, "stress concentration" can be formed around the nanoparticles, causing the PF resin matrix to be yielded. Consequently, a much amount 
of energy of distortion is absorbed. In addition, due to the "anchor points" effect, the copper nanoparticles have good adhesion with the PF resin, preventing the cracks from propagating around the copper nanoparticles. Therefore, the toughness of PF resin is improved. As the number of copper nanoparticles increases, the toughening effects mentioned above increase, so the impact strength of the modified PF resin increases. But after the number of nanosized copper particles exceeds some critical value, due to the very close distance between the particles, the microcracks in the modified PF resin easily develop into macrocracks, thereby resulting in a decrease in the impact strength of the PF resin.

\section{CONCLUSIONS}

The PF resins modified by nanosized copper particles were successfully prepared using an in situ polymerization process. The copper nanoparticles in the PF modified resins are spherical in geometry with a measurement of $30-60 \mathrm{~nm}$ in diameter. They are dispersed well in the PF resin matrix.

The thermal stability of the PF resin is significantly improved by nanosized copper particles at the temperature below the $T_{\mathrm{d}}$. In comparison with the pure PF resin, the $T_{\mathrm{d}}$ of the modified one may increase by $47^{\circ} \mathrm{C}$. However, at elevated temperature above the $T_{\mathrm{d}}$, the copper nanoparticles increase the rate of the degradation of the PF resin. The effects of copper nanoparticles on the thermal properties of PF resin are needed in friction materials' application.

The toughening effect of copper nanoparticles on the PF resin is outstanding. The impact strength of the modified PF resin may increase by $66.6 \%$ when compared to the pure one.

\section{REFERENCES}

1. C. C. M. Ma, S. C. Sung, and F. Y. Wang, J. Polym. Sci. Part A: Polym. Chem., 39, 2436 (2001).

2. M. H. Choi, H. Y. Byun, and I. J. Chung, Polymer, 16, 4437 (2002).

3. M. Abdalla, A. Ludwich, and T. Mitchell, Polymer, 24, 7353 (2003).

4. S. Sarkar and B. Adhikari, Polymer, 4, 518 (2001).

5. M. H. Choi, I. J. Chung, and J. D. Lee, Chem. Mater., 12, 2977 (2000).

6. R. H. Lin, L. Fang, Y. X. Xi, and Y. X. Shao, Acta Chim. Sinica, 23, 2365 (2004).

7. H. P. Klug and L. E. Alexander, "X-ray diffraction Procedures for Polycrystalline and Amorphous Materials," 2nd ed., Wiley, New York, N.Y., 1974.

8. F. R. Huang and Y. S. Jiao, "Phenolic Resins and Their Applications," Chemical Industry Press, Peking, 2003.

9. L. Costa, D. M. L. Rossi, G. Camino, E. D. Weill, and E. M. Pearce, Polym. Degrad. Stab., 56, 23 (1997).

10. Z. K. Zhang, Z. L. Cui, K. Z. Chen, Y. N. Wang, and Y. P. Ning, Chin. Sci. Bull., 20, 1852 (1995).

11. K. Monika, P. Filip, Z. Weiss, and R. Peter, Polym. Degrad. Stab., 84, 49 (2004). 